\title{
ABOUT THE LINK BETWEEN THE DETAILED DESCRIPTION OF TRANSITIONS IN AN ION AND THE AVERAGE-ION MODELS*
}

\author{
GUIDO CAVALLARO ${ }^{\dagger}$, LAURENT DESVILLETTES ${ }^{\ddagger}$, AND VALERIA RICCI§
}

\begin{abstract}
We study the link which exists between microscopic (detailed) models for the evolution of the electronic configurations in a population of ions and the macroscopic (average ion) models. Rigorous asymptotics are presented in situations where they exist (large temperature; almost empty or almost full shells), and numerical simulations are presented.
\end{abstract}

Key words. Average-ion models, microscopic models, rigorous asymptotics, comparison of solutions.

AMS subject classifications. Primary: 35Q40, 82D10, 37M05. Secondary: 34C11.

\section{Introduction}

In the last few years, the extension of average-ion models to the modeling of plasmas in off-equilibrium conditions has been considered (cf. $[5,3])$. The validity of this class of models, which are meant to give a simplified macroscopic statistical description of the state of a large set of ions as an alternative to the more complex detailed description based on evolution equations associated to microscopic processes in the plasma, is in general justified on the basis of heuristic arguments, since a priori the average-ion models can strictly be used only when the local thermodynamic equilibrium approximation is valid.

In this paper we study the link connecting the microscopic detailed description of a set of ions and its average-ion description in off-equilibrium conditions through the analysis of a toy model involving only simple processes in the plasma. The analysis can be considered somehow complementary to the analysis performed in papers dealing with the validation problem for linear Boltzmann type equations (like $[9,2]$ or $[6]$, just to give a very short list), where the goal is to find a correct simplified description for a set of particles, which in our case would be the sea of free particles (electrons or photons) surrounding the ions. We shall give for our toy model some rigorous (asymptotic) equivalence results and we shall present numerical simulations.

In what follows, we shall consider a set of ions which belong to the same species of atoms in a bath of particles (electrons) at Maxwellian equilibrium at a given temperature $T$. We denote by $Z$ the charge of the nucleus of the considered atomic species.

We consider the set of bound electrons in each ion and we collect the electrons in subsets which we shall call levels.

Levels are defined by grouping electrons with about the same energy, and usually the grouping is built in such a way that the number of levels $N$ for bound electrons is finite. In our simulations, the levels will be indexed according to the principal quantum number $n$ (up to the number $N$ which is a priori fixed), so that they will

*Received: January 8, 2009; accepted: March 25, 2009. Communicated by Francois Golse.

†Dipartimento di Matematica, Università di Roma "La Sapienza", P.le A. Moro 2, 00185 Rome, ITALY + ENS Cachan, CMLA, CNRS, PRES UniverSud, 61, Av. du Pdt. Wilson, 94235 Cachan Cedex, FRANCE (cavallaro@mat.uniroma1.it).

$\ddagger$ ENS Cachan, CMLA, CNRS \& IUF, PRES UniverSud, 61, Av. du Pdt. Wilson, 94235 Cachan Cedex, FRANCE (desville@cmla.ens-cachan.fr).

$\S$ Dipartimento di Metodi e Modelli Matematici, Università di Palermo, Viale delle Scienze Edificio 8, I90128 Palermo, ITALY (ricci@unipa.it, ricci@cmla.ens-cachan.fr). 
correspond to the atomic shells, and we shall use indifferently both words (shells or levels) to denote the same object.

A configuration $\vec{k}=\left(k_{1}, \ldots, k_{N}\right)$ of an ion is specified by the occupation number (i.e. the number of electrons) $k_{i} \in \mathbb{N}$ of each level $i$ in the ionic configuration.

Each bound electron shell can accommodate a finite number of electrons; we shall denote by $D_{i}$ the maximal number of electrons which can be accommodated in the shell $i\left(D_{i}=2 i^{2}\right.$ in the numerical example that we present). We shall denote as $\mathcal{C}$ the set of all ionic configurations $\vec{k}$.

Electrons can switch their energy to a value corresponding to a different level (bound-bound transitions), and be expelled or absorbed by the ion (continuum-bound transitions). We shall include this last kind of transition by modeling the set of free-electrons as the $N+1$-st shell; we write then $D_{N+1}=\infty$ for coherence.

At what we shall call the microscopic level, the set of ions is described by the probability to find an ion in the configuration $\vec{k}$ at time $t$, which we denote by $g_{\vec{k}}(t)$. We have of course $\sum_{\vec{k}} g_{\vec{k}}(t)=1$, and the evolution equation for $g_{\vec{k}}(t)$ is:

$$
\partial_{t} g_{\vec{k}}(t)=\sum_{\vec{k}^{\prime} \in \mathcal{C}} B_{\vec{k}^{\prime} \rightarrow \vec{k}} g_{\vec{k}^{\prime}}(t)-\sum_{\vec{k}^{\prime} \in \mathcal{C}} B_{\vec{k} \rightarrow \vec{k}^{\prime}} g_{\vec{k}}(t)
$$

where $B_{\vec{k}^{\prime} \rightarrow \vec{k}}$ is the rate of the transition $\vec{k}^{\prime} \rightarrow \vec{k}$. We notice that, because we included among the allowed transitions the processes of ionization and recombination, the transition $\vec{k}^{\prime} \rightarrow \vec{k}$ does not necessarily preserve the total number of electrons in the configuration $\vec{k}$.

The description of the system can be simplified thanks to the use of a macroscopic model in which the set of ions in different electronic configurations is replaced by a set of ions all in the same electronic configuration (average ions). The electronic configuration of each (and every) ion in this last system is such that the occupation number of each shell of the average ion is the average of the occupation numbers of the corresponding shell of the ions in the original system.

At this macroscopic level, the set of ions is described by the collection of populations of levels for the average ion, which we shall denote by $\left\{P_{h}\right\}_{h \geq 1}$ or $\vec{P}=\left(P_{1}, \ldots, P_{N}\right)$, where $P_{h} \in\left[0, D_{h}\right]$ denotes the (non necessarily integer) population of the $h$-th level of the average ion. In this model, given the level populations $\vec{P}$ at any time $t$, the probability $G_{\vec{k}}$ to find an ion in the configuration $\vec{k}$ is then computed as

$$
G_{\vec{k}}=\prod_{h=1}^{N}\left(\begin{array}{l}
D_{h} \\
k_{h}
\end{array}\right)\left(\frac{P_{h}}{D_{h}}\right)^{k_{h}}\left(1-\frac{P_{h}}{D_{h}}\right)^{D_{h}-k_{h}},
$$

as if ions would be in what we shall call a local equilibrium.

In the average ion description, $\vec{P}$ satisfies the following evolution equation (for $n=1, \ldots, N)$ :

$$
\frac{d}{d t} P_{n}=A_{n}\left(\left\{P_{m}\right\}_{m \geq 1, \neq n}\right)\left(1-\frac{P_{n}}{D_{n}}\right)-B_{n}\left(\left\{P_{m}\right\}_{m \geq 1, \neq n}\right) P_{n}
$$

where we denote by $A_{n}$ the total transition rate to the level $n$ from other levels (including the continuum) and by $B_{n}$ the total transition rate to other levels (including the continuum) from level $n$. In general, the rates $A_{n}$ and $B_{n}$ are functions of the population of the levels in the form of a sum of coefficients (themselves depending 
on $\vec{P})$ multiplied by $P_{m}$ or $\left(1-P_{m} / D_{m}\right)$ for $m \neq n$ plus a term coming from the ionization-recombination processes.

We would like to get an equation of the form (1.3) as a consequence (in a certain asymptotics) of an evolution equation for the probability $g_{\vec{k}}$ of the form (1.1), analogous (even though not strictly in the same sense) to the reduction of hierarchies of equations which describe many particle systems to a single equation for a one particle density (cf. for instance [4]). To this purpose, we shall compare the evolution of the populations of the shells $\vec{f}(t)=\left(f_{1}(t), \ldots, f_{N}(t)\right)$ defined for $h=1, \ldots, N$ by

$$
f_{h}(t)=\sum_{\vec{k} \in \mathcal{C}} k_{h} g_{\vec{k}}(t)
$$

where $g_{\vec{k}}$ satisfies equation (1.1), with the evolution of $\vec{P}$, the solution of equation (1.3).

In order to keep things as simple as possible, we consider the evolution of the level populations in ions where the only transition processes between levels involved are excitation and de-excitation (concerning only one bound electron) which are due to collisions with particles in the bath (including the continuum-bound transitions which may change the total number of electrons in an ionic configuration). As a consequence, we do not take into account the radiative transitions (cf. [8] for the physics of such transitions, and for example [1] for a mathematical study of the radiative transfer equations) and the two-electron collisional transitions. In section 2, we describe in detail the microscopic model that we shall study, and in Section 3 we write a non closed equation for the populations $\vec{f}$ of the shells. This equation can be closed under a factorization assumption which is related to the equilibrium. In section 4 we rigorously study the asymptotics which enable passage from the macroscopic model to the average ion model. Numerical illustrations are then provided in section 5 .

\section{Definition of the microscopic model}

When needed, we shall use the following notation for sums of vectors:

$$
\vec{k}+(h, l)_{i j}=\left\{\begin{array}{lc}
\left(k_{1}, \ldots, k_{i}+h, \ldots, k_{j}+l, \ldots, k_{N}\right) & 1 \leq i<j \leq N \\
\left(k_{1}, \ldots, k_{i}+h, \ldots, k_{N}\right) & 1 \leq i \leq N, j=N+1 .
\end{array}\right.
$$

Given the assumption on the kind of transitions we are going to consider, the transition probability $B_{\vec{k} \rightarrow \vec{k}^{\prime}}$ will be nonzero only when $\vec{k}^{\prime}=\vec{k}+( \pm 1, \mp 1)_{i j}$, for some $i, j \in\{1, \ldots, N\}$.

We shall now describe the quantities and the rates of transition corresponding to our microscopic model, which is built in order to be as close as possible to the macroscopic atomic model in [5].

2.1. Effective charges and energy levels. With respect to the choice of the effective charge $Z_{n}^{*}$, which models the part of the nucleus charge effectively interacting with an electron in the $n$-th shell, we shall analyze the following (two) different models:

- In the first model (macroscopically screened model), we shall assume

$$
Z_{n}^{*}=Z_{n}^{*}(\vec{f}) .
$$

With this choice the screening is not a microscopic quantity, since it does not correspond to a single atom. It has the advantage that it makes the 
comparison between microscopic and macroscopic evolutions easier in a way that we shall make explicit later on.

- In the second model (microscopically screened model), we shall assume

$$
Z_{n}^{*}=Z_{n}^{*}(\vec{k}) .
$$

Here, the screening due to an electron in the $h$-th shell on an electron in the $n$-th shell is taken into account in each atom independently.

We shall then consider the energy levels corrected by the screening effect defined above:

$$
E_{n}=E_{n}\left(Z_{n}^{*}\right)
$$

The effective charge of the ion $Z^{*}$ is given by the formula

$$
Z^{*}=Z^{*}(\vec{f})=Z-\sum_{h=1}^{N} f_{h}
$$

We avoid choosing a microscopic dependence in the effective charge $Z^{*}$ (although this would be in principle possible) because this is rarely used in the applications.

We shall denote in general the effective charge of the ion by $Z^{*}$, and sometimes $Z^{*}(\vec{f})$, when we wish to stress the dependence with respect to $\vec{f}$.

2.2. Transition probabilities and transition rates. In our first model, we assume that (for $n \neq m$ and $\vec{k}^{\prime}=\vec{k}+( \pm 1, \mp 1)_{\min (n, m), \max (n, m)}$ ),

$$
B_{\vec{k} \rightarrow \vec{k}^{\prime}}=R_{n m}^{c} k_{n}\left(1-\frac{k_{m}}{D_{m}}\right)
$$

where $R_{n m}^{c}$ depends on $(m, n), E_{n}-E_{m}$ and $T$. Here, $\left(E_{m}\right)_{m=1, . ., N}$ is function (through $Z_{n}^{*}$ ) of $\vec{f}$ (cf. equations (2.3) and (2.1)), and $T$ is the temperature of the bath.

The microscopic transition probability $R_{n m}^{c} \geq 0$ is associated to the excitation process when $n<m$ or to its inverse process (de-excitation) when $n>m$, and to the ionization process when $m=N+1$ or to its inverse process (recombination) when $n=$ $N+1$. For the continuum-bound, bound-continuum transitions, we define $k_{N+1}=Z^{*}$, $D_{N+1}=\infty, E_{N+1}=0$.

As a consequence of the detailed balance principle, the microscopic transition probabilities satisfy the following conditions: for $n<m, n, m=1, \ldots, N$,

$$
R_{m n}^{c}=\frac{D_{n}}{D_{m}} e^{\frac{E_{n}-E_{m}}{T}} R_{n m}^{c}
$$

and for $n=1, \ldots, N$,

$$
R_{N+1 n}^{c}=D_{n} C_{T} e^{\frac{E_{n}}{T}} R_{n N+1}^{c},
$$

where $C_{T}$ is a positive constant (depending only on $T$ ).

In our second model, we assume the microscopic transition probabilities to be of the form (2.5), where $R_{n m}^{c}$ depends on $(m, n), E_{n}(\vec{k})-E_{m}\left(\vec{k}^{\prime}\right)$, and $T$. We write $R_{n m}^{c}\left(\vec{k}, \vec{k}^{\prime}\right)$ for the sake of simplicity in the following. 
As a consequence of the detailed balance principle, the microscopic transition probabilities satisfy the following conditions: for $n<m, n, m=1, \ldots, N$,

$$
R_{m n}^{c}\left(\vec{k}, \vec{k}+(1,-1)_{n m}\right)=\frac{D_{n}}{D_{m}} e^{\frac{E_{n}\left(\vec{k}+(1,-1)_{n m}\right)-E_{m}(\vec{k})}{T}} R_{n m}^{c}\left(\vec{k}+(1,-1)_{n m}, \vec{k}\right),
$$

and for $n=1, \ldots, N$,

$$
R_{N+1 n}^{c}(\vec{k})=D_{n} C_{T} e^{\frac{E_{n}\left(\vec{k}+(1,-1)_{n N+1}\right)}{T}} R_{n N+1}^{c}\left(\vec{k}+(1,-1)_{n N+1}\right),
$$

where $C_{T}$ is the positive constant which also appeared in the first model.

2.3. Evolution microscopic equations. According to these transition probabilities, we can write down the evolution equation (for our different models) of the probability $g_{\vec{k}}(t)$.

We begin with our first model:

$$
\begin{aligned}
\partial_{t} g_{\vec{k}}= & \sum_{j=1}^{N} \sum_{m=j+1}^{N}\left[\left(k_{j}+1\right)\left(1-\frac{k_{m}-1}{D_{m}}\right) 1_{\left\{k_{j} \neq D_{j}, k_{m} \neq 0\right\}} g_{\vec{k}+(1,-1)_{j m}}\right. \\
& \left.-k_{m}\left(1-\frac{k_{j}}{D_{j}}\right) \frac{D_{j}}{D_{m}} e^{\frac{E_{j}-E_{m}}{T}} g_{\vec{k}}\right] \times R_{j m}^{c} \\
& +\sum_{m=1}^{N} \sum_{j=m+1}^{N}\left[\left(k_{j}+1\right)\left(1-\frac{k_{m}-1}{D_{m}}\right) \frac{D_{m}}{D_{j}} e^{-\frac{E_{j}-E_{m}}{T}} 1_{\left\{k_{j} \neq D_{j}, k_{m} \neq 0\right\}} g_{\vec{k}+(-1,1)_{m j}}\right. \\
& \left.-k_{m}\left(1-\frac{k_{j}}{D_{j}}\right) g_{\vec{k}}\right] \times R_{m j}^{c} \\
& +\sum_{j=1}^{N}\left[\left(k_{j}+1\right) 1_{k_{j} \neq D_{j}} g_{\vec{k}+(1,-1)_{j N+1}}\right. \\
& \left.-Z^{*}(\vec{f}) C_{T}\left(1-\frac{k_{j}}{D_{j}}\right) D_{j} e^{\frac{E_{j}}{T}} g_{\vec{k}}\right] R_{j N+1}^{c} \\
& +\sum_{j=1}^{N}\left[Z^{*}(\vec{f}) C_{T}\left(1-\frac{k_{j}-1}{D_{j}}\right) D_{j} e^{\frac{E_{j}}{T}} 1_{k_{j} \neq 0} g_{\vec{k}+(-1,1)_{j N+1}}-k_{j} g_{\vec{k}}\right] R_{j N+1}^{c} \cdot(2.10)
\end{aligned}
$$

We recall that in this formula, the rates $R_{j m}^{c}$, etc., depend on the energy levels $E_{j}$. Those levels depend on $\vec{f}$ through equation (2.1) and (2.3).

Then, we write the corresponding formula for our second model:

$$
\begin{aligned}
\partial_{t} g_{\vec{k}}= & \sum_{j=1}^{N} \sum_{m=j+1}^{N}\left[\left(k_{j}+1\right)\left(1-\frac{k_{m}-1}{D_{m}}\right) 1_{\left\{k_{j} \neq D_{j}, k_{m} \neq 0\right\}} g_{\vec{k}+(1,-1)_{j m}}\right. \\
& \left.-k_{m}\left(1-\frac{k_{j}}{D_{j}}\right) \frac{D_{j}}{D_{m}} e^{\frac{E_{j}\left(\vec{k}+(1,-1)_{j m}\right)-E_{m}(\vec{k})}{T}} g_{\vec{k}}\right] \times R_{j m}^{c}\left(\vec{k}+(1,-1)_{j m}, \vec{k}\right) \\
& +\sum_{m=1}^{N} \sum_{j=m+1}^{N}\left[\left(k_{j}+1\right)\left(1-\frac{k_{m}-1}{D_{m}}\right) \frac{D_{m}}{D_{j}} e^{-\frac{E_{j}\left(\vec{k}+(-1,1)_{m j}\right)-E_{m}(\vec{k})}{T}} 1_{\left\{k_{j} \neq D_{j}, k_{m} \neq 0\right\}} g_{\vec{k}+(-1,1)_{m j}}\right. \\
& \left.-k_{m}\left(1-\frac{k_{j}}{D_{j}}\right) g_{\vec{k}}\right] \times R_{m j}^{c}\left(\vec{k}, \vec{k}+(-1,1)_{m j}\right) \\
& +\sum_{j=1}^{N}\left[\left(k_{j}+1\right) 1_{k_{j} \neq D_{j}} g_{\vec{k}+(1,-1)_{j N+1}}\right.
\end{aligned}
$$




$$
\begin{aligned}
& \left.-Z^{*}(\vec{f}) C_{T}\left(1-\frac{k_{j}}{D_{j}}\right) D_{j} e^{\frac{E_{j}\left(\vec{k}+(1,-1)_{j N+1}\right)}{T}} g_{\vec{k}}\right] R_{j N+1}^{c}\left(\vec{k}+(1,-1)_{j N+1}\right) \\
& +\sum_{j=1}^{N}\left[Z^{*}(\vec{f}) C_{T}\left(1-\frac{k_{j}-1}{D_{j}}\right) D_{j} e^{\frac{E_{j}(\vec{k})}{T}} 1_{k_{j} \neq 0} g_{\vec{k}+(-1,1)_{j N+1}}-k_{j} g_{\vec{k}}\right] R_{j N+1}^{c}(\vec{k})(2.1)
\end{aligned}
$$

3. Reduction of the microscopic equations to macroscopic equations and their closure

3.1. Non closed equations. When we consider our first model, it is possible to write a simplified (non closed) equation for the quantities $f_{h}$, starting from equation (2.10) and making a suitable change of indices, by summing over all possible configurations. It reads as

$$
\begin{aligned}
\frac{d}{d t} f_{h}= & \sum_{j=1}^{h-1} \sum_{\vec{k}}\left[k_{j}\left(1-\frac{k_{h}}{D_{h}}\right)-k_{h}\left(1-\frac{k_{j}}{D_{j}}\right) \frac{D_{j}}{D_{h}} e^{\left.\frac{E_{j}(\vec{f})-E_{h}(\vec{f})}{T}\right] g_{\vec{k}} R_{j h}^{c}(\vec{f})}\right. \\
& +\sum_{j=h+1}^{N} \sum_{\vec{k}}\left[k_{j}\left(1-\frac{k_{h}}{D_{h}}\right) \frac{D_{h}}{D_{j}} e^{-\frac{E_{j}(\vec{f})-E_{h}(\vec{f})}{T}}-k_{h}\left(1-\frac{k_{j}}{D_{j}}\right)\right] g_{\vec{k}} R_{h j}^{c}(\vec{f}) \\
& +\sum_{\vec{k}}\left[Z^{*}(\vec{f}) C_{T}\left(1-\frac{k_{h}}{D_{h}}\right) D_{h} e^{\frac{E_{h}(\vec{f})}{T}}-k_{h}\right] g_{\vec{k}} R_{h N+1}^{c}(\vec{f}) .
\end{aligned}
$$

The corresponding equation for our second model is

$$
\begin{aligned}
\frac{d}{d t} f_{h}= & \sum_{j=1}^{h-1} \sum_{\vec{k}}\left[k_{j}\left(1-\frac{k_{h}}{D_{h}}\right) R_{j h}^{c}\left(\vec{k}, \vec{k}+(-1,1)_{j h}\right)\right. \\
& \left.-k_{h}\left(1-\frac{k_{j}}{D_{j}}\right) \frac{D_{j}}{D_{h}} e^{\frac{E_{j}\left(\vec{k}+(1,-1)_{j h}\right)-E_{h}(\vec{k})}{T}} R_{j h}^{c}\left(\vec{k}+(1,-1)_{j h}, \vec{k}\right)\right] g_{\vec{k}} \\
& +\sum_{j=h+1}^{N} \sum_{\vec{k}}\left[k_{j}\left(1-\frac{k_{h}}{D_{h}}\right) \frac{D_{h}}{D_{j}} e^{\frac{E_{h}\left(\vec{k}+(1,-1)_{h j}\right)-E_{j}(\vec{k})}{T}} R_{h j}^{c}\left(\vec{k}+(1,-1)_{h j}, \vec{k}\right)\right. \\
& \left.-k_{h}\left(1-\frac{k_{j}}{D_{j}}\right) R_{h j}^{c}\left(\vec{k}, \vec{k}+(-1,1)_{h j}\right)\right] g_{\vec{k}} \\
& +\sum_{\vec{k}}\left[Z^{*}(\vec{f}) C_{T}\left(1-\frac{k_{h}}{D_{h}}\right) D_{h} e^{\frac{E_{h}\left(\vec{k}+(1,-1)_{h N+1)}\right.}{T}} R_{h N+1}^{c}\left(\vec{k}+(1,-1)_{h N+1}\right)\right. \\
& \left.-k_{h} R_{h N+1}^{c}(\vec{k})\right] g_{\vec{k}} .
\end{aligned}
$$

3.2. Factorized solutions. In the case of our first model, if there exists a factorized solution of (2.11) on a certain interval of time, i.e., $g_{\vec{k}}(t)=\prod_{h=1}^{N} \hat{g}_{h}\left(k_{h}, t\right)$ (and of course $\sum_{k_{h}=1}^{D_{h}} \hat{g}_{h}\left(k_{h}, t\right)=1$ ), equation (3.1) becomes closed:

$$
\frac{d}{d t} P_{h}=\sum_{j=1}^{h-1}\left[P_{j}\left(1-\frac{P_{h}}{D_{h}}\right)-P_{h}\left(1-\frac{P_{j}}{D_{j}}\right) \frac{D_{j}}{D_{h}} e^{\frac{E_{j}(\vec{P})-E_{h}(\vec{P})}{T}}\right] R_{j h}^{c}(\vec{P})
$$




$$
\begin{aligned}
& +\sum_{j=h+1}^{N}\left[P_{j}\left(1-\frac{P_{h}}{D_{h}}\right) \frac{D_{h}}{D_{j}} e^{-\frac{E_{j}(\vec{P})-E_{h}(\vec{P})}{T}}-P_{h}\left(1-\frac{P_{j}}{D_{j}}\right)\right] R_{h j}^{c}(\vec{P}) \\
& +\left[Z^{*}(\vec{P}) C_{T}\left(1-\frac{P_{h}}{D_{h}}\right) D_{h} e^{\frac{E_{h}(\vec{P})}{T}}-P_{h}\right] R_{h N+1}^{c}(\vec{P}),
\end{aligned}
$$

where $P_{j}(t):=f_{j}(t)=\sum_{k_{j}=0}^{D_{j}} k_{j} \hat{g}_{j}\left(k_{j}, t\right)$. equation (3.3) coincides with equation (1.3) for the average population on the $h$-th level in the corresponding average-ion model, by defining $A$ and $B$ in a suitable way.

Notice that equation (3.3) cannot be obtained from a microscopic model (even when a factorized solution is assumed) when a microscopic screening is imposed (that is, for example, in the case of our second model). It also could not be obtained from a microscopic model in which the effective charge would be microscopic (that is, depending on $\vec{k}$ rather than $\vec{f})$.

3.3. Equilibrium. We now analyze the existence of equilibrium solutions for Equations (2.10), (2.11) and (3.3), and the connection between them.

Since this is the most relevant case in physical applications, we shall only look for equilibrium solutions which do not depend on the choice of the transition rates $R_{n m}^{c}$ : this corresponds to looking for probability densities $g_{\vec{k}}(t)$ or occupation numbers $P_{h}(t)$ such that each coefficient in the linear combination of transitions rates on the right-hand side of $(2.10),(2.11)$ or $(3.3)$ is identically equal to 0 .

In our second model, represented by equation (2.11), in order to satisfy all the constraints, compatibility conditions will be required.

3.3.1. Microscopic equilibrium. We shall first look for an equilibrium solution of equations (2.10) and (2.11).

Setting each coefficient of the linear combination of transitions rates on the righthand side of (2.11) equal to 0 , we get a system of equations having the following compatibility condition on the energy:

$$
E_{j}(\vec{k})-E_{j}\left(\vec{k}+(-1,1)_{s N+1}\right)=E_{s}(\vec{k})-E_{s}\left(\vec{k}+(-1,1)_{j N+1}\right)
$$

for $s \neq j$. Whenever this condition is fulfilled, we can obtain a (non necessarily factorized) microscopic equilibrium solution.

Assuming electron energies of the form presented in (5.2), condition (3.4) is unfortunately not satisfied for our second model.

For the first model (corresponding to equation (2.10)), the equilibrium (factorized) solution is:

$$
g_{\vec{k}}^{e q}=\prod_{h=1}^{N}\left(\begin{array}{c}
D_{h} \\
k_{h}
\end{array}\right) \frac{\left(Z^{*}\left(\vec{f}^{e q}\right) C_{T} e^{\frac{E_{h}\left(\vec{f}^{e q}\right)}{T}}\right)^{k_{h}}}{\left(1+Z^{*}(\vec{f} e q) C_{T} e^{\frac{E_{h}\left(\vec{f}^{e q}\right)}{T}}\right)^{D_{h}}} .
$$

Note that this solution is given in implicit form. We shall see that (3.5) can be connected in a simple way to the equilibrium solution of the macroscopic model.

3.3.2. Macroscopic equilibrium. Equation (3.3) has as equilibrium solution the Fermi-Dirac distribution, given (implicitly) by:

$$
P_{h}^{e q}=D_{h} \frac{Z^{*}\left(\vec{P}^{e q}\right) C_{T} e^{\frac{E_{h}\left(\vec{P}^{e q}\right)}{T}}}{1+Z^{*}\left(\vec{P}^{e q}\right) C_{T} e^{\frac{E_{h}\left(\vec{P}^{e q}\right)}{T}}} .
$$


When we consider the function

$$
g_{\vec{k}}^{e q}=\prod_{h=1}^{N}\left(\begin{array}{c}
D_{h} \\
k_{h}
\end{array}\right)\left(\frac{P_{h}^{e q}}{D_{h}}\right)^{k_{h}}\left(1-\frac{P_{h}^{e q}}{D_{h}}\right)^{D_{h}-k_{h}},
$$

where $P_{h}^{e q}$ is given by formula (3.6), we find that $g_{\vec{k}}^{e q}$ is given by (3.5). This means that for our first model the macroscopic equilibrium can be obtained as an average of the microscopic equilibrium. We therefore expect that for large times the result of the microscopic and macroscopic simulations coincide. This property is however not shared by our second model, as we shall see in the fourth simulation of section 5 .

One can verify that for our first model, the equilibrium solution is the only solution of the microscopic equations which remains factorized on an interval of time (that is, all other factorized initial data immediately lose the property of being factorized in the evolution of equation (2.10)), so that the closure presented in subsection 3.2 cannot be considered to be consistent. As a consequence, we can in general deduce the macroscopic equations from the microscopic ones only in some asymptotics that will be detailed in next section.

\section{Asymptotic analysis}

We begin with a proposition based mainly on Gronwall's lemma which enables us to make explicit the evolution of the difference between the microscopic and macroscopic descriptions (for our first model) in terms of its initial value, the correlation matrix $\sum_{\vec{k}} k_{h} k_{j} g_{\vec{k}}(s)-f_{h}(s) f_{j}(s)$, and the temperature $T$ of the bath.

Proposition 4.1. We consider $N \geq 2$, an integer number, $T>0$, and a family $\left(D_{i}\right)_{i=1, . ., N}$ of numbers of $\mathbb{N}$. We denote $\mathcal{D}=\max \left(D_{i}\right)$. We also consider a function $Z^{*}$ of $\vec{f}$ and a sequence $\left(E_{n}\right)_{n \in \mathbb{N}}$ of functions of $\vec{f}$ which all lie in $W^{1, \infty}$ (that is, the space of bounded and Lipschitz-continuous functions). Then, we take a family of transition rates $\left(R_{i j}^{c}\right)_{i=1, ., N ; j=i+1, . ., N+1}$ which are functions of $T$ and $\vec{f}$ and lie (for all $T$ ) in the space $W^{1, \infty}$ with respect to the variable $\vec{f}$. Finally, we take a constant $C_{T}>0$.

Then for all $T_{\text {inf }}>0$ and $t_{1}>0$, one can find a constant $K$ depending only on $N, \mathcal{D},\left\|Z^{*}\right\|_{W^{1, \infty}}, \sup _{n=1, . ., N}\left\|E_{n}\right\|_{W^{1, \infty}}, T_{\text {inf }}$ such that (for any $T \geq T_{\text {inf }}>0$ and $t \in$ $\left.\left[0, t_{1}\right]\right)$ if $\vec{P}:=\vec{P}(t)$ is solution of equation (3.3) and $\left(g_{\vec{k}}\right)_{\vec{k} \in \mathcal{C}}:=\left(g_{\vec{k}}\right)_{\vec{k} \in \mathcal{C}}(t)$ is solution of equation (2.10), then

$$
\begin{aligned}
& \sum_{h=1, \ldots, N}\left|f_{h}(t)-P_{h}(t)\right| \\
\leq & \left(\sum_{h=1, \ldots, N}\left|f_{h}(0)-P_{h}(0)\right|+K T^{-1} \sum_{j, h} \int_{0}^{t_{1}}\left|\sum_{\vec{k}} k_{h} k_{j} g_{\vec{k}}(s)-f_{h}(s) f_{j}(s)\right| d s\right) e^{K \varphi(T) t},
\end{aligned}
$$

where

$$
\varphi(T):=\sup _{h, j}\left\|R_{h j}^{c}(T, \cdot)\right\|_{W^{1, \infty}}+\left(1+C_{T}\right) \sup _{h}\left\|R_{h N+1}^{c}(T, \cdot)\right\|_{W^{1, \infty}}
$$

and $f_{h}$ is given (knowing $g_{\vec{k}}$ ) by formula (1.4). 
Proof. Since we are looking at our first model, we can write

$$
\begin{aligned}
\frac{d}{d t} f_{h}(t)= & \sum_{j=1}^{N} A_{h j}(\vec{f}) f_{j}(t) \\
& -\left[\sum_{j=1}^{N} A_{j h}(\vec{f})+R_{h N+1}^{c}(\vec{f})\left(Z^{*}(\vec{f}) C_{T} e^{\frac{E_{h}(\vec{f})}{T}}+1\right)\right] f_{h}(t) \\
& -\sum_{j=1}^{N} B_{h j}(\vec{f}) \chi_{h j}+R_{h N+1}^{c} Z^{*}(\vec{f}) C_{T} D_{h} e^{\frac{E_{h}(\vec{f})}{T}}
\end{aligned}
$$

and

$$
\begin{aligned}
\frac{d}{d t} P_{h}(t)= & \sum_{j=1}^{N} A_{h j}(\vec{P}) P_{j}(t) \\
& -\left[\sum_{j=1}^{N} A_{j h}(\vec{P})+R_{h N+1}^{c}(\vec{P})\left(Z^{*}(\vec{P}) C_{T} e^{\frac{E_{h}(\vec{P})}{T}}+1\right)\right] P_{h}(t) \\
& -\sum_{j=1}^{N} B_{h j}(\vec{P}) P_{h} P_{j}+R_{h N+1}^{c}(\vec{P}) Z^{*}(\vec{P}) C_{T} D_{h} e^{\frac{E_{h}(\vec{P})}{T}}
\end{aligned}
$$

where

$$
\begin{array}{r}
\chi_{h j}(t)=\sum_{\vec{k}} k_{h} k_{j} g_{\vec{k}}(t), \\
A_{h j}= \begin{cases}R_{j h}^{c} & j<h \\
R_{h j}^{c} \frac{D_{h}}{D_{j}} e^{-\frac{E_{j}-E_{h}}{T}} & j>h, \\
0 & j=h\end{cases} \\
B_{h j}=\frac{\left(1-e^{\frac{E_{j}-E_{h}}{T}}\right)}{D_{h}} A_{h j},
\end{array}
$$

and the evolution of the quantity $f_{h}-P_{h}$ is given by

$$
\begin{aligned}
\frac{d}{d t}\left(f_{h}(t)-P_{h}(t)\right)= & \sum_{j=1}^{N}\left[A_{h j}(\vec{f}) f_{j}(t)-A_{h j}(\vec{P}) P_{j}(t)\right] \\
& -\left\{\left[\sum_{j=1}^{N} A_{j h}(\vec{f})+R_{h N+1}^{c}(\vec{f})\left(Z^{*}(\vec{f}) C_{T} e^{\frac{E_{h}(\vec{f})}{T}}+1\right)\right] f_{h}(t)\right. \\
& \left.-\left[\sum_{j=1}^{N} A_{j h}(\vec{P})+R_{h N+1}^{c}(\vec{P})\left(Z^{*}(\vec{P}) C_{T} e^{\frac{E_{h}(\vec{P})}{T}}+1\right)\right] P_{h}(t)\right\} \\
& -\sum_{j=1}^{N}\left[B_{h j}(\vec{f}) \chi_{h j}(t)-B_{h j}(\vec{P}) P_{h} P_{j}\right] \\
& +C_{T} D_{h}\left[R_{h N+1}^{c}(\vec{f}) e^{\frac{E_{h}(\vec{f})}{T}} Z^{*}(\vec{f})-R_{h N+1}^{c}(\vec{P}) e^{\frac{E_{h}(\vec{P})}{T}} Z^{*}(\vec{P})\right]
\end{aligned}
$$


We can rewrite (4.5) as

$$
\begin{aligned}
& \frac{d}{d t}\left(f_{h}(t)-P_{h}(t)\right) \\
= & \sum_{j=1}^{N} A_{h j}(\vec{f})\left(f_{j}(t)-P_{j}(t)\right) \\
& -\left[\sum_{j=1}^{N} A_{j h}(\vec{f})+R_{h N+1}^{c}(\vec{f})\left(Z^{*}(\vec{f}) C e^{\frac{E_{h}(\vec{f})}{T}}+1\right)\right]\left(f_{h}(t)-P_{h}(t)\right) \\
& -\sum_{j=1}^{N} B_{h j}(\vec{f})\left(\chi_{h j}(t)-P_{h} P_{j}\right) \\
& +\sum_{j=1}^{N}\left[A_{h j}(\vec{f})-A_{h j}(\vec{P})\right] P_{j}(t) \\
& +\left\{\sum_{j=1}^{N}\left[A_{j h}(\vec{P})-A_{j h}(\vec{f})\right]\right. \\
& \left.+\left[R_{h N+1}^{c}(\vec{P})\left(Z^{*}(\vec{P}) C_{T} e^{\frac{E_{h}(\vec{P})}{T}}+1\right)-R_{h N+1}^{c}(\vec{f})\left(Z^{*}(\vec{f}) C_{T} e^{\frac{E_{h}(\vec{f})}{T}}+1\right)\right]\right\} P_{h}(t) \\
& +\sum_{j=1}^{N}\left[B_{h j}(\vec{P})-B_{h j}(\vec{f})\right] P_{h} P_{j}+C_{T} D_{h}\left[R_{h N+1}^{c}(\vec{f}) e^{\frac{E_{h}(\vec{f})}{T}} Z^{*}(\vec{f})-R_{h N+1}^{c}(\vec{P}) e^{\frac{E_{h}(\vec{P})}{T}} Z^{*}(\vec{P})\right] .
\end{aligned}
$$

Therefore,

$$
\begin{aligned}
\sum_{h}\left|f_{h}(t)-P_{h}(t)\right| \leq & \sum_{h}\left|f_{h}(0)-P_{h}(0)\right|+2 N \sup _{h, j}\left\|A_{h j}(T, \cdot)\right\|_{\infty} \int_{0}^{t} \sum_{j}\left|f_{j}(s)-P_{j}(s)\right| d s \\
& +\sup _{h}\left\|R_{h N+1}^{c}(T, \cdot)\left(Z^{*} C_{T} e^{\frac{E_{h}}{T}}+1\right)\right\|_{\infty} \int_{0}^{t} \sum_{j}\left|f_{j}(s)-P_{j}(s)\right| d s \\
& +\sup _{h, j}\left\|B_{h j}(T, \cdot)\right\|_{\infty} \sum_{j, h=1}^{N} \int_{0}^{t}\left|\sum_{\vec{k}}\left[k_{h} k_{j}-f_{h}(s) f_{j}(s)\right] g_{\vec{k}}(s)\right| d s \\
& +2 N \mathcal{D} \sup _{h, j}\left\|A_{h j}(T, \cdot)\right\|_{L i p} \int_{0}^{t} \sum_{j}\left|f_{j}(s)-P_{j}(s)\right| d s \\
& +\mathcal{D} \sup _{h}\left\|R_{h N+1}^{c}(T, \cdot)\left(Z^{*} C_{T} e^{\frac{E_{h}}{T}}+1\right)\right\|_{L i p} \int_{0}^{t} \sum_{j}\left|f_{j}(s)-P_{j}(s)\right| d s \\
& +N \mathcal{D}^{2} \sup _{h, j}\left\|B_{h j}(T, \cdot)\right\|_{L i p} \int_{0}^{t} \sum_{j}\left|f_{j}(s)-P_{j}(s)\right| d s \\
& +\mathcal{D} \sup _{h}\left\|R_{h N+1}^{c}(T, \cdot) Z^{*} C_{T} e^{\frac{E_{h}}{T}}\right\|_{L i p} \int_{0}^{t} \sum_{j}\left|f_{j}(s)-P_{j}(s)\right| d s,
\end{aligned}
$$

so that

$$
\sum_{h}\left|f_{h}(t)-P_{h}(t)\right| \leq \sum_{h}\left|f_{h}(0)-P_{h}(0)\right|+\left(2 N \mathcal{D} \sup _{h, j}\left\|A_{h j}(T, \cdot)\right\|_{W^{1, \infty}}\right.
$$




$$
\begin{aligned}
& +\underset{h}{ } \sup _{h}\left\|R_{h N+1}^{c}(T, \cdot) Z^{*} C_{T} e^{\frac{E_{h}}{T}}\right\|_{W^{1, \infty}}+\mathcal{D} \sup _{h}\left\|R_{h N+1}^{c}(T, \cdot)\right\|_{W^{1, \infty}} \\
& \left.+N \mathcal{D}^{2} \sup _{h, j}\left\|B_{h j}(T, \cdot)\right\|_{L i p}\right) \int_{0}^{t} \sum_{j}\left|f_{j}(s)-P_{j}(s)\right| d s \\
& +\sup _{h, j}\left\|B_{h j}(T, \cdot)\right\|_{\infty} \sum_{j, h=1}^{N} \int_{0}^{t}\left|\sum_{\vec{k}}\left[k_{h} k_{j}-f_{h}(s) f_{j}(s)\right] g_{\vec{k}}(s)\right| d s .
\end{aligned}
$$

Then, we observe that

$$
\begin{aligned}
& \sup _{h, j}\left\|A_{h j}(T, \cdot)\right\|_{W^{1, \infty}} \leq \mathcal{D} \sup _{h, j}\left\|R_{h j}^{c}(T, \cdot)\right\|_{W^{1, \infty}} e^{\sup _{h} \frac{\left\|E_{h}\right\|_{\infty}}{T_{i n f}}}\left(1+2 \frac{\sup _{h}\left\|E_{h}\right\|_{L i p}}{T_{i n f}}\right), \\
& \left\|B_{h j}(T, \cdot)\right\|_{\infty} \leq 2 \frac{\sup _{h}\left\|E_{h}\right\|_{\infty}}{T} e^{2 \sup _{h} \frac{\left\|E_{h}\right\|_{\infty}}{T_{i n f}}}, \\
& \quad\left\|B_{h j}(T, \cdot)\right\|_{L i p} \\
& \leq 2\left\|A_{h j}(T, \cdot)\right\|_{W^{1, \infty}} \frac{\sup _{h}\left\|E_{h}\right\|_{W^{1, \infty}}}{T} e^{\sup _{h} \frac{\left\|E_{h}\right\|_{\infty}}{T_{i n f}}} \\
& \leq 2 \mathcal{D} \sup _{h, j}\left\|R_{h j}^{c}(T, \cdot)\right\|_{W^{1, \infty}} \frac{\sup _{h}\left\|E_{h}\right\|_{W^{1, \infty}}}{T} e^{2 \sup _{h} \frac{\left\|E_{h}\right\|_{\infty}}{T_{i n f}}}\left(1+2 \frac{\sup _{h}\left\|E_{h}\right\|_{L i p}}{T_{i n f}}\right), \\
& \quad\left\|R_{h N+1}^{c}(T, \cdot) Z^{*} C_{T} e^{\frac{E_{h}}{T}}\right\|_{W^{1, \infty}} \leq C_{T}\left\|Z^{*}\right\|_{W^{1, \infty}}\left\|R_{h N+1}^{c}(T, \cdot)\right\|_{W^{1, \infty}} \\
& \quad \times\left(1+\frac{\sup _{h}\left\|E_{h}\right\|_{L i p}}{T_{i n f}}\right) e^{\sup _{h} \frac{\left\|E_{h}\right\|_{\infty}}{T_{i n f}}} .
\end{aligned}
$$

As a consequence, for some constant $K$ which depends only on $N, \mathcal{D}, T_{\text {inf }},\left\|Z^{*}\right\|_{W^{1, \infty}}$ and $\sup _{h}|| E_{h} \|_{W^{1, \infty}}$,

$$
\begin{aligned}
& \sum_{h}\left|f_{h}(t)-P_{h}(t)\right| \leq \sum_{h}\left|f_{h}(0)-P_{h}(0)\right| \\
& +K \varphi(T) \int_{0}^{t} \sum_{j}\left|f_{j}(s)-P_{j}(s)\right| d s+K T^{-1} \sum_{j, h=1}^{N} \int_{0}^{t}\left|\sum_{\vec{k}}\left[k_{h} k_{j}-f_{h}(s) f_{j}(s)\right] g_{\vec{k}}(s)\right| d s,
\end{aligned}
$$

where

$$
\varphi(T)=\sup _{h, j}\left\|R_{h j}^{c}(T, \cdot)\right\|_{W^{1, \infty}}+\left(1+C_{T}\right)\left\|R_{h N+1}^{c}(T, \cdot)\right\|_{W^{1, \infty}} .
$$

Then, thanks to Gronwall's lemma (and for all $t \in\left[0, t_{1}\right]$ ):

$$
\begin{aligned}
& \sum_{h}\left|f_{h}(t)-P_{h}(t)\right| \\
\leq & \left(\sum_{h}\left|f_{h}(0)-P_{h}(0)\right|+K T^{-1} \sum_{j, h=1}^{N} \int_{0}^{t_{1}}\left|\sum_{\vec{k}}\left[k_{h} k_{j}-f_{h}(s) f_{j}(s)\right] g_{\vec{k}}(s)\right| d s\right) e^{K \varphi(T) t},
\end{aligned}
$$

and Proposition 4.1 is proven.

We can deduce from this proposition the equivalence of the microscopic and macroscopic descriptions when the temperature is large, under conditions which are satisfied by the rates (which are those of [5]) taken in the simulations of section 5 . 
COROllary 4.2 (High Temperature limit). Under the same assumption as in Proposition 4.1, and for initial data such that $P_{h}(0)=f_{h}(0)$ (that is, "well prepared" initial data), $h=1, \ldots, N$, if

$$
\begin{array}{r}
\lim _{T \rightarrow \infty} \sup _{n, m}\left\|R_{n m}^{c}(T, \cdot)\right\|_{W^{1, \infty}}<+\infty \\
\lim _{T \rightarrow \infty} C_{T} \sup _{n}\left\|R_{n N+1}^{c}(T, \cdot)\right\|_{W^{1, \infty}}<+\infty,
\end{array}
$$

then, for large $T$ and for any $t_{1}>0$

$$
\sup _{t \in\left[0, t_{1}\right]} \sum_{j=1}^{N}\left|f_{j}(t)-P_{j}(t)\right|=O\left(T^{-1}\right) .
$$

Proof. Since Proposition 4.1 is valid and $f_{h}(0)=P_{h}(0)$ for all $h$,

$$
\sum_{j=1}^{N}\left|f_{j}(t)-P_{j}(t)\right| \leq N^{2} \mathcal{D}^{2} K T^{-1} t_{1} e^{K \varphi(T) t},
$$

where $\varphi$ is given by (4.1), so that, from (4.7) and (4.8), we get (4.9). This ends the proof of Corollary 4.2 .

REMARK 4.3. Though we also have (thanks to the analysis of equilibria in section 3) $\lim _{t \rightarrow \infty} \sum_{j}\left|f_{j}(t)-P_{j}(t)\right|=0$, it doesn't seem possible to take $t_{1}=+\infty$ in estimate (4.9).

We now turn to another type of asymptotics, namely the situation in which all shells are almost full or almost empty during the evolution of the plasma.

COROLlary 4.4. Under the assumptions of Proposition 4.1, if, for all $t \in\left[0, t_{1}\right]$, $\frac{f_{h_{i}}(t)}{D_{h_{i}}}<\varepsilon$, for $i=1, \ldots, N_{1}$ and $1-\frac{f_{h_{i}}(t)}{D_{h_{i}}}<\varepsilon$ for $i=N_{1}+1, \ldots, N$ (for some $N_{1} \in$ $\{1, . ., N\})$, and if $\sum_{h=1, \ldots, N}\left|f_{h}(0)-P_{h}(0)\right| \leq \mathcal{C}_{1} \varepsilon$, then for all $t \in\left[0, t_{1}\right]$,

$$
\sum_{h=1}^{N}\left|f_{h}(t)-P_{h}(t)\right| \leq \mathcal{C}_{2} \varepsilon
$$

where

$$
\mathcal{C}_{2}=\left(\mathcal{C}_{1}+N^{2} K T^{-1} \mathcal{D}^{3} t_{1}\right) e^{K \varphi(T) t},
$$

and $K, \varphi$ are the constants appearing in Proposition 4.1 (and formula (4.1)).

Proof. We first observe that under our assumption, for $i=1, \ldots, N_{1}$ (and any $\delta \in$ ] $0, D_{h_{i}}[)$,

$$
\sum_{\vec{k}: k_{h_{i}} \geq \delta} g_{\vec{k}}(t) \leq \frac{D_{h_{i}}}{\delta} \varepsilon
$$

and for $i=N_{1}+1, . ., N$,

$$
\sum_{\vec{k}: k_{h_{i}} \leq D_{h_{i}}-\delta} g_{\vec{k}}(t) \leq \frac{D_{h_{i}}}{\delta} \varepsilon
$$


Then, for $t \in\left[0, t_{1}\right], h, j=1, . ., N$,

$$
\begin{aligned}
\sum_{\vec{k}}\left(k_{h} k_{j}-f_{h}(t) f_{j}(t)\right) g_{\vec{k}}(t)= & 1_{h \in\left\{1, . ., N_{1}\right\}} \sum_{k_{j_{h}} \geq 1}\left(k_{j}-f_{j}\right) k_{j_{h}} g_{\vec{k}}(t) \\
& +1_{h \in\left\{N_{1}+1, . ., N\right\}} \sum_{k_{j_{h}} \leq D_{j_{h}}-1}\left(k_{j}-f_{j}\right)\left(k_{j_{h}}-D_{j_{h}}\right) g_{\vec{k}}(t),
\end{aligned}
$$

so that thanks to the estimate above,

$$
\left|\sum_{\vec{k}}\left(k_{h} k_{j}-f_{h}(t) f_{j}(t)\right) g_{\vec{k}}(t)\right| \leq \mathcal{D}^{3} \varepsilon
$$

From (4.1) and (4.13) we then obtain

$$
\sum_{h=1, \ldots, N}\left|f_{h}(t)-P_{h}(t)\right| \leq\left(\sum_{h=1, \ldots, N}\left|f_{h}(0)-P_{h}(0)\right|+N^{2} K T^{-1} \mathcal{D}^{3} \varepsilon t_{1}\right) e^{K \varphi(T) t} .
$$

This ends the proof of Corollary 4.4.

It is of course difficult to guarantee that the shells will remain almost full or almost empty on a long interval of time, it is however at least possible to show that for a small interval of time, it remains so if it is true initially. This is the point of the following proposition:

Proposition 4.5. Under the same hypothesis as in Proposition 4.1, and for initial data such that $P_{h}(0)=f_{h}(0), h=1, \ldots, N$ (that is, "well prepared" initial data), if

$$
\begin{aligned}
\frac{P_{h_{i}}(0)}{D_{h_{i}}}<\varepsilon & \text { for } & i=1, \ldots, N_{1} \\
1-\frac{P_{h_{i}}(0)}{D_{h_{i}}}<\varepsilon & \text { for } & i=N_{1}+1, \ldots, N
\end{aligned}
$$

for some $N_{1} \in\{1, . ., N\}$, then

$$
\sum_{h=1}^{N}\left|f_{h}(t)-P_{h}(t)\right| \leq \mathcal{C}\left(\varepsilon t+t^{2}\right) e^{K \varphi(T) t},
$$

where $K$ and $\varphi$ are the constants appearing in Proposition 4.1 (and formula (4.1)), and the constant $\mathcal{C}$ depends on $T, \sup _{n, m}\left\|R_{n m}^{c}(T, \cdot)\right\|_{W^{1, \infty}},\|Z\|_{W^{1, \infty}}, N, \sup _{h}\left\|E_{h}\right\|_{W^{1, \infty}}$ and $\mathcal{D}$.

REMARK 4.6. We can in particular consider as initial datum $g_{\vec{k}}(0)$ the microscopic equilibrium solution (3.7) for a temperature $T^{*}$ such that for each $P_{h}^{e q}$, either $\frac{P_{h}^{e q}\left(T^{*}\right)}{D_{h}}<$ $\varepsilon$ or $1-\frac{P_{h}\left(T^{*}\right)}{D_{h}}<\varepsilon$, with $\varepsilon$ small. This is what is done in the two first simulations of section 5 .

\section{Proof of Proposition 4.5.}

Proof. We have for the correlation matrix (for any $h, j=1, . ., N$ ): 


$$
\begin{aligned}
& \left|\sum_{\vec{k}}\left(k_{h} k_{j}-f_{h}(t) f_{j}(t)\right) g_{\vec{k}}(t)-\sum_{\vec{k}}\left(k_{h} k_{j}-f_{h}(0) f_{j}(0)\right) g_{\vec{k}}(0)\right| \\
\leq & t \sup _{s \in[0, t]}\left|\sum_{\vec{k}}\left(k_{h} k_{j}-f_{h}(s) f_{j}(s)\right) \dot{g}_{\vec{k}}(s)-\left(\dot{f}_{h}(s) f_{j}(s)+\dot{f}_{j}(s) f_{h}(s)\right) g_{\vec{k}}(s)\right| \\
\leq & \sup _{n, m}\left\|R_{n m}^{c}(T)\right\|_{\infty}\left[4 \mathcal{D}^{2} N(1+N \mathcal{D}) e^{\frac{\sup _{h}\left\|E_{h}\right\|_{\infty}}{T}}\right. \\
& \left.+\left(4 N \mathcal{D}^{3}+2 \mathcal{D}^{2}\right)\left(1+\left\|Z^{*}\right\|_{\infty} C_{T} e^{\frac{\sup _{h}\left\|E_{h}\right\|_{\infty}}{T}}\right)\right] t \\
:= & c_{1} t
\end{aligned}
$$

and $c_{1}$ depends on $\sup _{n, m}\left\|R_{n m}^{c}(T, \cdot)\right\|_{\infty}, T,\left\|Z^{*}\right\|_{\infty}, \mathcal{D}, N, \sup _{h}\left\|E_{h}\right\|_{\infty}$.

According to the proof of Corollary 4.4, for $h=1, \ldots, N$,

$$
\left|\sum_{\vec{k}}\left(k_{h} k_{j}-f_{h}(0) f_{j}(0)\right) g_{\vec{k}}(0)\right| \leq \mathcal{D}^{3} \varepsilon .
$$

Therefore, we obtain the bound

$$
\int_{0}^{t}\left|\sum_{\vec{k}}\left(k_{h} k_{j}-f_{h}(t) f_{j}(s)\right) g_{\vec{k}}(s)\right| d s \leq \mathcal{D}^{3} \varepsilon t+\frac{c_{1}}{2} t^{2} .
$$

We use then Proposition 4.1 to obtain

$$
\sum_{h=1}^{N}\left|f_{h}(t)-P_{h}(t)\right| \leq K T^{-1} N^{2}\left(\mathcal{D}^{3} \varepsilon t+\frac{c_{1}}{2} t^{2}\right) e^{K \varphi(T) t} .
$$

Finally, Proposition 4.5 is proven.

Remark 4.7. Of course, both Corollary 4.4 and Proposition 4.5 are valid (with obvious changes in the proof) when all shells are almost empty $\left(\frac{P_{i}}{D_{i}}, \frac{f_{i}}{D_{i}}<\varepsilon\right.$ for $i=$ $1, \ldots, N)$ or all shells are almost full $\left(1-\frac{P_{i}}{D_{i}}, 1-\frac{f_{i}}{D_{i}}<\varepsilon\right.$ for $\left.i=1, \ldots, N\right)$.

\section{Numerical simulations}

In this section we present some figures in order to illustrate the rigorous results of the previous section. For each figure, we represent (for some $h$ ) the functions $f_{h}$ (obtained by solving the microscopic equation (2.10) or (2.11)) and $P_{h}$ (obtained by solving the macroscopic equation (3.3)).

The results have been obtained using a standard second order explicit scheme for ODEs (note that for the microscopic model, the number of ODEs to solve is very large: 16929).

Here are the values of the functions and parameters used in the simulations.

The shells are built according to the first quantum number, so that $D_{n}=2 n^{2}$. Only $N=4$ shells (plus the continuum) are introduced, so that the computation time remains reasonable.

The screening effect (that, is, the effective charge of the nucleus seen by an electron) is modeled by

$$
Z_{n}^{*}=\left\{\begin{array}{l}
Z-\sum_{h<n} f_{h}-\frac{1}{3} f_{n} \text { first (macro }- \text { screened) model } \\
Z-\sum_{h<n} k_{h}-\frac{1}{3} k_{n} \text { second (micro }- \text { screened) model. }
\end{array}\right.
$$




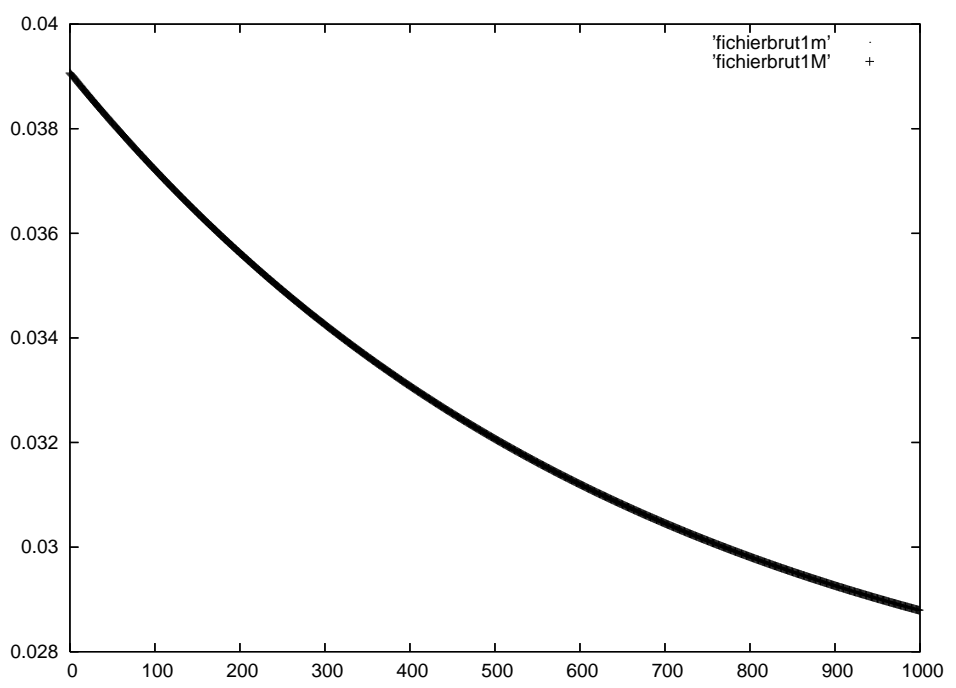

FIG. 5.1. Occupation numbers $f_{1}(t)$ and $P_{1}(t)$ for our first model with $T_{0}=4.3 k e V$ and $T=$ $4.5 \mathrm{keV}$. The curve 'fichierbrut1m' corresponds to $f_{1}(t)$ while the curve 'fichierbrut1 $M$ ' corresponds to $P_{1}(t)$.

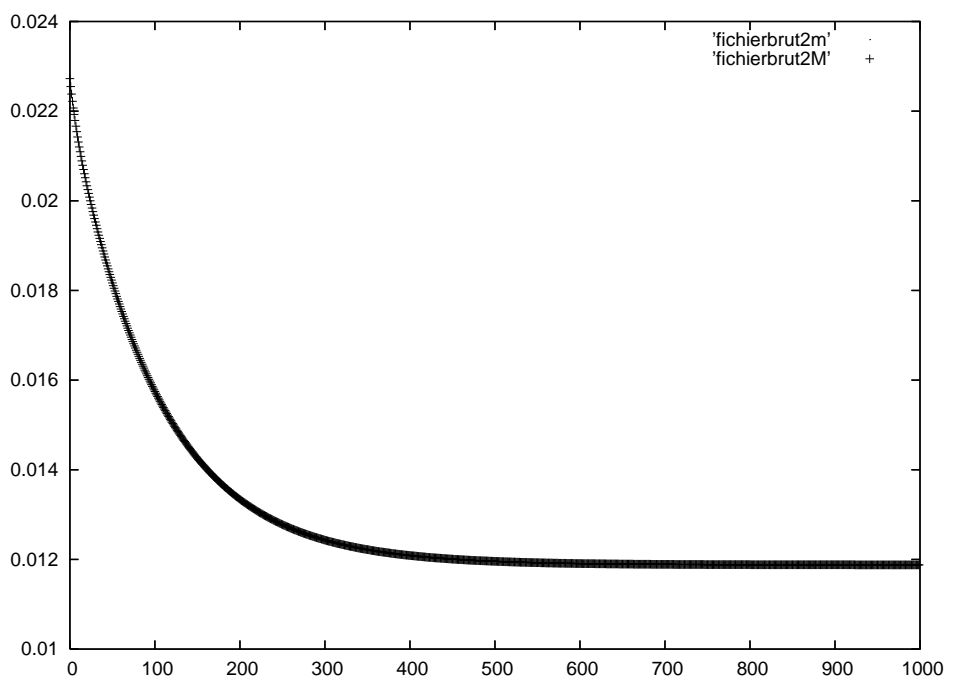

FIG. 5.2. Occupation numbers $f_{2}(t)$ and $P_{2}(t)$ for our first model with $T_{0}=1.7 \mathrm{keV}$ and $T=$ $1.9 \mathrm{keV}$. The curve 'fichierbrut2m' corresponds to $f_{2}(t)$ while the curve 'fichierbrut2M' corresponds to $P_{2}(t)$.

Then the energy of each level is that of the hydrogenic atom corrected by the screening effect defined above:

$$
E_{n}=0.0136 \frac{\left(Z_{n}^{*}\right)^{2}}{n^{2}} k e V
$$




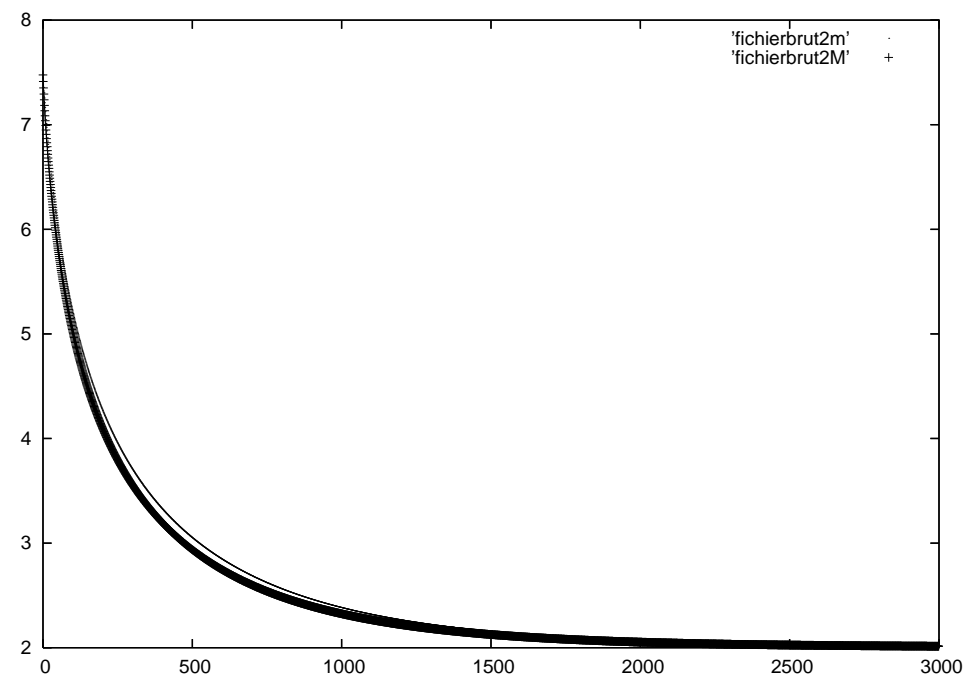

FIG. 5.3. Occupation numbers $f_{2}(t)$ and $P_{2}(t)$ for our first model with $T_{0}=0.6 \mathrm{keV}$ and $T=$ $0.9 \mathrm{keV}$. The curve 'fichierbrut2m' corresponds to $f_{2}(t)$ while the curve 'fichierbrut2M' corresponds to $P_{2}(t)$.

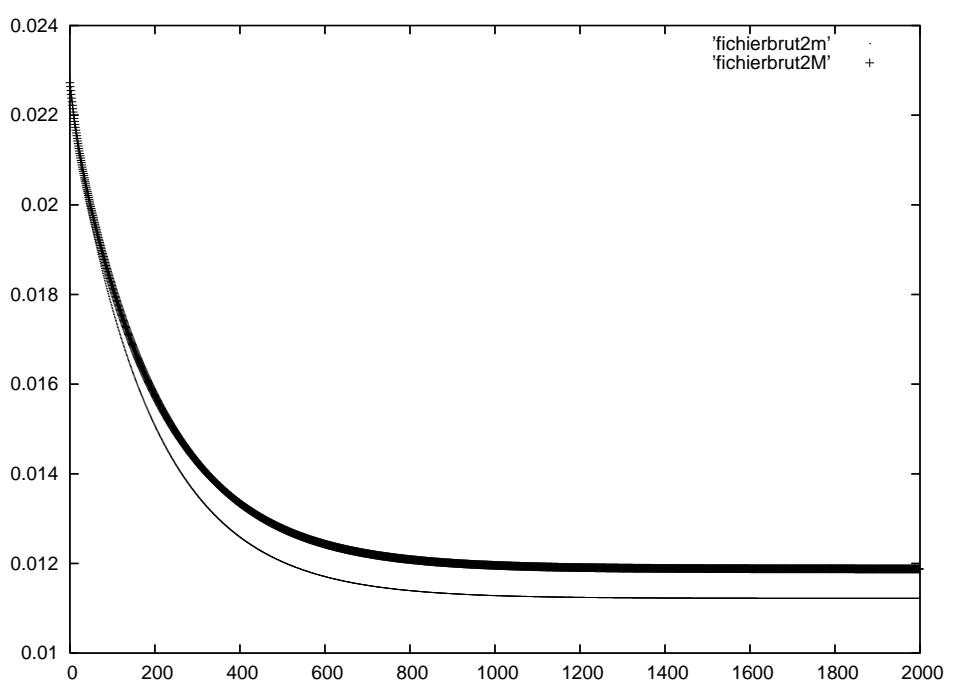

FIG. 5.4. Occupation numbers $f_{2}(t)$ and $P_{2}(t)$ for our third model with $T_{0}=1.7 \mathrm{keV}$ and $T=$ $1.9 \mathrm{keV}$. The curve 'fichierbrut2m' corresponds to $f_{2}(t)$ while the curve 'fichierbrut2M' corresponds to $P_{2}(t)$.

The rates of transition (for $n<m$ ) are given in our first model by

$$
\begin{aligned}
R_{n m}^{c} & =\frac{\mathcal{R}_{n m}}{E_{n}-E_{m}} e^{-\frac{E_{n}-E_{m}}{T}}, \\
R_{n N+1}^{c} & =\mathcal{R}_{n N+1} \frac{\left[1-e^{\left.-\frac{E_{n}}{T}\right]}\right.}{E_{n}^{2}} e^{-\frac{E_{n}}{T}},
\end{aligned}
$$


and in our second model by

$$
\begin{aligned}
R_{n m}^{c}\left(\vec{k}, \vec{k}+(-1,1)_{n m}\right) & =\frac{\mathcal{R}_{n m}}{E_{n}(\vec{k})-E_{m}\left(\vec{k}+(-1,1)_{n m}\right)} e^{-\frac{E_{n}(\vec{k})-E_{m}(\vec{k}+(-1,1) n m)}{T}}, \\
R_{n m}^{c}\left(\vec{k}+(1,-1)_{n m}, \vec{k}\right) & =\frac{\mathcal{R}_{n m}}{E_{n}\left(\vec{k}+(1,-1)_{n m}\right)-E_{m}(\vec{k})} e^{-\frac{E_{n}\left(\vec{k}+(1,-1)_{n m}\right)-E_{m}(\vec{k})}{T}}, \\
R_{n N+1}^{c}(\vec{k}) & =\mathcal{R}_{n N+1} \frac{\left[1-e^{\left.-\frac{E_{n}(\vec{k})}{T}\right]}\right.}{E_{n}^{2}(\vec{k})} e^{-\frac{E_{n}(\vec{k})}{T}}, \\
\left.R_{n N+1}^{c}\left(\vec{k}+(1,-1)_{n N+1}\right)\right) & =\mathcal{R}_{n N+1} \frac{\left[1-e^{-\frac{\left.E_{n}\left(\vec{k}+(1,-1)_{n N+1}\right)\right)}{T}}\right]}{\left.E_{n}^{2}\left(\vec{k}+(1,-1)_{n N+1}\right)\right)} e^{-\frac{E_{n}\left(\vec{k}+(1,-1)_{n N+1}\right)}{T}} .
\end{aligned}
$$

In those formulas, we have used the following values for $\mathcal{R}_{n m}$ (taken from [5]):

$$
\begin{aligned}
\mathcal{R}_{n m} & =\frac{4.99 \times 10^{-10} f(n, m) g_{n m} N_{e}}{\sqrt{T}}, \\
\mathcal{R}_{n N+1} & =3.45 \times 10^{-11} N_{e} \sqrt{T} \Gamma_{n},
\end{aligned}
$$

with the Gaunt factor $g_{n m}=0.361$, and the values $f(1,2)=0.4161, f(1,3)=0.0792$, $f(1,4)=0.029, f(2,3)=0.637, f(2,4)=0.119, f(3,4)=0.8408$. Moreover, we take

$$
\Gamma_{n}=2.8014 e^{-\frac{n}{n+5}},
$$

and for the electron number density:

$$
N_{e}=\frac{6.02 \times 10^{23} \rho}{M} Z^{*}
$$

where $\rho$ is the plasma mass density (taken as $5 \times 10^{-2} \mathrm{gcm}^{-3}$ ), $Z^{*}$ is given by formula (2.4), $Z$ is the atomic number of the atom (taken as 50) and $M$ is the mass number of the atom (taken as 120). Note that the value of $Z$ is such that all the denominators appearing in the rates $R_{n m}^{c}$ defined above are nonzero (and are in fact bigger than a strictly positive constant).

Finally, the constant $C_{T}$ appearing in the process of ionization is taken to be

$$
C_{T}=\frac{\rho}{317 M T^{\frac{3}{2}}},
$$

and the temperature $T$ of the bath (in $k e V$ ) is chosen in a different way for the different numerical simulations.

In all simulations, we take as initial datum the formulas (3.6), (3.7), at a given temperature $T_{0}$, which differs from the temperature $T$ of the bath.

We begin by showing a figure corresponding to the case $T_{0}=4.3 \mathrm{keV}$ and $T=$ $4.5 \mathrm{keV}$, for our first model. This range of temperature is quite high: the levels are all almost empty. We show the evolution of the occupation numbers for the first shell, its order of magnitude is $10^{-2}$. As can be seen on the figure, the curves for $f_{1}(t)$ and $P_{1}(t)$ are indistinguishable: we are in the regime where we can apply Corollary 4.2 , which deals with the high temperature asymptotics.

Next, we present a figure corresponding to the case $T_{0}=1.7 \mathrm{keV}$ and $T=1.9 \mathrm{keV}$, for our first model. In this temperature range, the first shell is almost full while the 
other shells are almost empty. We show the evolution of the occupation numbers for the second shell, its order of magnitude is $10^{-1}-10^{-2}$. The curves for $f_{2}(t)$ and $P_{2}(t)$ are once again indistinguishable: we are in the regime where we can apply Corollary 4.4, which deals with shells which are almost empty or almost full. One can also compute the correlations appearing between the occupation numbers of different shells: along the evolution, they never grow over orders of magnitude of $10^{-7}$.

Our third figure corresponds to the case $T_{0}=0.6 \mathrm{keV}$ and $T=0.9 \mathrm{keV}$, for our first model. In this temperature range, the occupation number for the second shell is far from 0 and $D_{2}=8$. As a consequence, the evolution of $f_{2}$ differs somewhat (as can be seen on the graph) from the evolution of $P_{2}$. Correlations involving the second shell (in particular $\chi_{23}-P_{2} P_{3}$ ) grow up to order of $10^{-3}$.

Finally, we show a figure corresponding to our second model (that is, with the microscopic screening), for $T_{0}=1.7 \mathrm{keV}$ and $T=1.9 \mathrm{keV}$ (those are the same temperatures as that of Fig. 5.2). We see that (especially for large times), the curves $f_{2}(t)$ and $P_{2}(t)$ are becoming different: this is due to the fact that there is no microscopic equilibrium in this case which is compatible with the macroscopic equilibrium.

Acknowledgments. G.C. was supported by a post-doctoral grant of Région Île de France (March 2007- February 2008). V. R. was partially supported by the european research group GREFI-MEFI in the early stage of this work.

D. Bouche, A. Decoster and F. Golse are warmly thanked for fruitful discussions during the preparation of this work.

\section{REFERENCES}

[1] C. Bardos, F. Golse, B. Perthame and R. Sentis, The nonaccretive radiative transfer equation: existence of solutions and Rosseland approximation, J. Funct. An., 77(2), 434-460, 1988.

[2] J. Bourgain, F. Golse and B. Wennberg, On the distribution of free path lengths for the periodic Lorentz gas, Commun. Math. Phys., 190, 491-508, 1998.

[3] P. Dallot, G. Faussurier, A. Decoster and A. Mirone, Average-ion level-population correlations in off-equilibrium plasmas, Phys. Rev. E, 57, 1017-1028, 1998.

[4] A. Decoster, P.A. Markowich and B. Perthame, Modeling of Collisions, Series in Applied Mathematics (Paris) 2, Éditions Scientifique et Médicales Elsevier Paris - North Holland Amsterdam, 1998.

[5] A. Djaoui, S.J. Rose, Calculation of the time-dependent excitation and ionization in a laserproduced plasma, J. Phys. B: At. Mol. Opt. Phys., 25, 2745-2762, 1992.

[6] F. Golse, On the periodic Lorentz gas and the Lorentz kinetic equation, Ann. Fac. Sci. Toulouse, in press.

[7] R.M. More, J. Quant. Spectroscop. Radiat. Transfer, 27, 345, 1982.

[8] D. Mihalas and B. Mihalas, Foundations of Radiations Hydrodynamics, Oxford University Press, 1984, Reprint Dover, 1999.

[9] H. Spohn, The Lorentz process converges to a random flight process, Commun. Math. Phys., 60(3), 277-290, 1978. 